\title{
A organização espacial condiciona as relações entre ambiente e saúde: o exemplo da exposição ao mercúrio em uma fábrica de lâmpadas fluorescentes
}

\author{
Environment and health relationships are \\ conditioned by the spatial organization: the case \\ of mercury exposure in a fluorescent lamp plant
}

Christovam Barcellos 1

Jorge M. Huet Machado 2

\footnotetext{
1 Departamento de Informações para a Saúde, Centro de Informação Científica e Tecnológica, Fundação Oswaldo Cruz. Av. Brasil 4365, Rio de Janeiro, RJ, 21045-900, Brasil. xris@fiocruz.br

2 Coordenação de Saúde do Trabalhador, Diretoria de Recursos Humanos, Fundação Oswaldo Cruz. Av. Brasil 4365,

Rio de Janeiro, RJ, 21045-900, Brasil.
}

Abstract Space has been used for the analyses relating environment and health, as simple geometric plan for the display of epidemiological data, as a proxy for the differentiation of social conditions, or as a circumstance of spatial factors inducing risk. In this work, the three main spatial approaches of health are described, as well as their theoretical limitations. The use geoprocessing in health has been facilitated due to the wide access to epidemiological data bases and the availability of computerized cartographic and statistical tools. However, the use of these tools presupposes models for the explanation of the health/disease process, that are based on a set of spatial variables such as distance, neighborhood, and the linkage of data for characterization of the place. The example of a factory of fluorescent lamps allows to understand the space as mediator of social relations. The location and activity in the factory impose the risk conditions to which the workers are submitted. The spatial analysis of mercury emission, contamination and exposure indicators, identified areas and activities that produce risk conditions to workers. These factors are also related to intern and external production organization.

Key words Environmental Epidemiology; Spatial Analysis; Mercury Exposure
Resumo O espaço tem sido utilizado nas análises que relacionam o ambiente com a saúde, ora como simples plano geométrico para a disposição de dados epidemiológicos, ora como uma aproximação para a diferenciação de condições sociais, ou mesmo como uma circunstância de fatores espaciais que induzem risco. Neste trabalho são descritas as três principais abordagens espaciais em saúde e suas limitações. $O$ uso do geoprocessamento na área de saúde tem sido facilitado pelo amplo acesso a bases de dados epidemiológicos e pela disponibilização de ferramentas cartográficas e estatísticas computadorizadas. O uso desta ferramenta pressupõe, no entanto, modelos de explicação do processo saúde/doença, que são baseados em variáveis espaciais como a distância, vizinhança, e o interrelacionamento com dados de caracterização do lugar. O exemplo de uma fábrica de lâmpadas fluorescentes permite compreender o espaço como mediador de relações sociais. A localização e atividade dos trabalhadores na fábrica condicionam os riscos a que estes estarão submetidos. Através da análise espacial de indicadores de emissão, contaminação e exposição ao mercúrio, foi possível identificar áreas e atividades de risco aos trabalhadores. Estes fatores, por sua vez, estão intrinsecamente ligados à organização interna e externa da produção. Palavras-chave Epidemiologia Ambiental; Análise Espacial; Exposição ao Mercúrio 


\section{Espaço e saúde: uma pequena introdução}

As relações entre o ambiente e a saúde têm sido estudadas, desde a antigüidade, através de diferentes abordagens cuja evolução foi paralela e no mesmo sentido do desenvolvimento da própria geografia. $\mathrm{O}$ uso do espaço como categoria de análise tem sido ressaltado em trabalhos recentes nas áreas de epidemiologia, geografia médica e análise ambiental.

Segundo Breilh (1991) a epidemiologia deve estudar os processos de mediação do meio geográfico e da reprodução social das classes com a finalidade de recuperar a unidade da realidade e entender os determinantes de seu movimento. O objetivo principal da geografia médica é elucidar as causas e os fatores que condicionam os padrões de distribuição geográfica das doenças e das condições que afetam os índices de saúde e de vida (Avila-Pires, 1983). Padrões espaciais de doenças podem permitir a identificação de fontes comuns de contaminação, trajetos influenciados por variáveis ambientais, padrões ou diferenças de situações de saúde ante perspectivas de agregações particulares em diversos níveis, bem como o planejamento e avaliação de intervenções e fatores sócio-econômicos espacializados que afetam perfis de saúde.

A geografia pode contribuir para a um aprofundamento dos estudos que relacionam ambiente e saúde através do uso de mapas e análise espacial para definir condições de representatividade de pontos de amostragem, na elaboração de critérios para o estabelecimento de regiões ou unidades espaciais de análise, modos e processos de difusão espacial de um evento de saúde, lugar e modos de vida dos indivíduos numa combinação de escalas espaciais (Picheral \& Salem, 1992).

Uma vez que grande parte das variáveis indicadoras da qualidade de vida, saúde e ambiente é localizável no espaço, o geoprocessamento se impõe como ferramenta de organização e análise de dados, particularmente através de uma de suas vertentes, o Sistema de Informações Geográficas (SIG) que pode ser definido a partir de três propriedades: a capacidade de apresentação cartográfica de informações complexas, uma sofisticada base integrada de objetos espaciais e de seus atributos ou dados, e um engenho analítico formado por um conjunto de procedimentos e ferramentas de análise espacial (Maguirre apud Castro, 1996). O crescente uso do geoprocessamento na área da saúde tem aumentado a capacidade de formular e avaliar hipóteses sobre a distribuição espacial de doenças e mortes, principalmente através da confecção rápida de mapas temáticos. A interpretação adequada desses mapas depende, no entanto, de uma concepção prévia do processo saúde/doença e do próprio espaço.

Este trabalho visa indicar os usos e limitações da categoria espaço como mediadora das relações entre sociedade, saúde e ambiente. Para isso, são analisadas três formas de utilização do espaço nas análises de saúde e discutido o exemplo de uma fábrica de lâmpadas, onde o arranjo espacial desempenha o papel de organização das relações de produção e divisão desigual do trabalho e dos riscos à saúde. O uso do espaço como categoria de análise tem sido reforçado em trabalhos que relacionam ambiente e saúde através de três principais vertentes descritas a seguir.

\section{O espaço - geometria}

O espaço pode ser compreendido como um conjunto de relações geométricas entre objetos num plano cartesiano. A premissa desta abordagem é de que os elementos espaciais próximos compartilham condições sócio-ambientais semelhantes. Este espaço-geometria permitiria identificar similaridades entre elementos próximos, diminuindo a "dependência espacial" de elementos à medida em que a distância entre eles aumenta. Uma grande diversidade de técnicas estatísticas têm sido desenvolvidas para incorporar a localização como uma variável condicionante do comportamento de um determinado fenômeno no espaço. Parte destas técnicas têm sido utilizadas na geoquímica visando identificar a direcionalidade e dispersão de veios de minérios em um terreno, a partir de amostras pontuais, de modo a indicar formas econômicas de exploração mineral. Estas técnicas, chamadas geoestatísticas, que permitem interpolação de dados, partem do princípio de que este espaço é um meio contínuo. Outras técnicas de análise estatística espacial permitem estabelecer a priori condições e critérios para o estudo de relações entre elementos geográficos, criando-se uma estrutura de conexões que identificam padrões de vizinhança entre localidades (Szwarcwald e Leal, 1997). Estas técnicas permitem incorporar a existência de barreiras e assimetrias nas relações entre elementos do espaço. 
Um exemplo da abordagem estatística na saúde ambiental são os levantamentos de ocorrência de leucemia próximos a usinas nucleares (Hills \& Alexander, 1989). Neste caso, procura-se certificar a validade de hipóteses de indução de doenças através de padrões de distribuição de casos relacionados às fontes de risco pré-estabelecidas. A distância da fonte suspeita de contaminação é utilizada como um parâmetro estimativo da exposição humana a agentes de risco. Esta estimativa é particularmente útil nos estudos ecológicos em que se deseja avaliar doses a que foram submetidos grupos humanos em longos períodos de tempo (Briggs, 1992). É importante lembrar, neste caso, que a exposição ou dose presumida é inversamente proporcional ao quadrado da distância à fonte suspeita. Este é também o caso da exposição a fontes de radiação ionizantes e de baixa freqüência, no qual o cálculo de distâncias entre fontes de emissão e população exposta permite estimar a dose de radiação a que esta população está potencialmente submetida (Wartenberg et al., 1993). Os padrões de deposição de poluentes em torno de fontes de emissão seguem também esta tendência, condicionados ainda pela direção dos ventos e pela presença de barreiras de relêvo (Godin et al., 1985).

A abordagem estatística espacial é também utilizada para o estudo de difusão espacial de doenças ou agentes infecciosos. Neste caso, os dados de saúde são separados em períodos de tempo de modo a analisar ou simular seqüencialmente quadros evolutivos das epidemias (Bailey e Gatrell, 1995; Werneck e Struchiner, 1997). Através desta técnica pode-se identificar barreiras ou áreas mais vulneráveis para a difusão de doenças. O uso do geoprocessamento em ambiente de SIG, nesse caso, facilita a criação de cenários e o cálculo de estimativas.

A abordagem geoestatística tem limitações importantes quando aplicada à difusão de doenças e agentes patogênicos. Somente uma parte desta difusão é realizada por contigüidade, isto é, com maiores possibilidades de "contágio" para elementos próximos. Um outro importante componente do processo de difusão é a presença de redes de troca, que não necessariamente obedecem critérios de contigüidade, e sim de hierarquia entre elementos espaciais (Hagget, 1975). Este é não só o caso da difusão de agentes infecciosos, e das migrações, mas também da difusão tecnológica, que alcança primeiramente cidades com nível hie- rárquico superior, como as metrópoles, para posteriormente chegar a locais mais próximos e com fluxos de troca menos intensos. Esta forma de dispersão tende a aumentar com a intensificação dos fluxos de pessoas e mercadorias. Os exemplos da dispersão da cólera no mundo (Hagget, 1975) e da AIDS no Brasil (Barcellos e Bastos, 1996a) confirmam a existência de nós (geograficamente identificáveis) que se comunicam e distam por vezes centenas de quilômetros, facilitando a difusão de agentes infecciosos. A partir da instalação deste agente infecioso no nó, ou sua colonização, este será exportado para outro nó ou servirá de base para a difusão para vizinhos hierarquicamente inferiores. Os processos de difusão necessariamente irão utilizar esta rede de trocas entre pessoas, lugares e cidades, envolvendo tanto o vizinho, quanto posições hierárquicas próximas. Um modelo de difusão espacial de doenças deverá, portanto, contar com ambos componentes.

Também nos estudos de padrões de distribuição de doenças no espaço, a distância, ou relação de vizinhança, não podem ser tomadas como principais explicadores dos valores observados entre vizinhos num mapa. O desenvolvimento humano sobre a superfície terrestre produz um espaço diferenciado, que viabiliza e intermedia a relação da sociedade com a natureza. Através do processo de desenvolvimento, são gerados os lugares dos pobres, das indústrias, dos ricos, de circulação, que são diferentes e próximos. Este processo produz, portanto, uma heterogenização do espaço, criando canais de troca entre estes lugares especializados. O espaço produzido será, como conseqüência, um mosaico de elementos e variáveis, que dificilmente se submeterão aos princípios geoestatísticos.

\section{O espaço - região}

Uma segunda concepção do espaço admite um mosaico de regiões com características próprias que não necessariamente estão relacionadas às dos seus vizinhos. A região, neste caso, pode tanto ser concebida como uma realidade concreta, construída ao longo de sua história, como ser objeto de criação intelectual a partir de parâmetros estatísticos que a caracterizem em função de objetivos preestabelecidos (Corrêa, 1986). Esta abordagem tem como principais pressupostos a homogeneidade interna e a independência das unidades 
espaciais de agregação e análise de dados (Barcellos e Bastos, 1996b; Openshawn et al., 1987).

Nos estudos de saúde ambiental, esta estratégia tem sido utilizada para a identificação de padrões de distribuição de doenças e seu relacionamento com fatores de risco ambiental, tais como condições de saneamento, habitação e poluição atmosférica. Neste caso, o padrão de distribuição da doença é desconhecido previamente e busca-se estatística ou visualmente sua identificação (Carvalho, 1997). Os dados epidemiológicos podem ser reagregados em unidades de análise baseado em critérios sócio-demográficos e administrativos. Esta abordagem, foi utilizada para estabelecer áreas de maior mortalidade infantil e relacioná-las aos possíveis fatores de risco na Região Metropolitana do Rio de Janeiro (Duchiade, 1991) e para estudar a mortalidade em áreas classificadas segundo características sócio-econômicas na Região Metropolitana de São Paulo (Stephens et al., 1994). O espaço, neste caso é concebido como produto e produtor de diferenciações sociais e ambientais. A análise multivariada de um conjunto de indicadores sócio-econômicos obtidos dos censos demográficos tem sido uma das formas mais utilizadas para a discriminação das regiões que formam este mosaico (Paim, 1997).

Outra forma de caracterização das regiões, com uso crescente na saúde pública, tem sido a identificação de habitats favoráveis para a proliferação de vetores através do sensoriamento remoto (Hugh-Jones, 1989). Esta linha de trabalho se aproxima dos princípios da teoria dos focos naturais de doenças, a teoria da nidalidade, desenvolvida por Pavlovsky, que relaciona a presença do "agente causal de uma enfermidade transmissível” a uma paisagem geográfica específica. Este habitat reúne um clima, uma vegetação, um solo onde vetores, hospedeiros e indivíduos suscetíveis se abrigam, incorporando a ecologia como ciência explicativa das relações entre meio interior e exterior na produção de doenças (Avila-Pires, 1983).

As limitações desta abordagem referem-se, principalmente à escolha da unidade a ser definida como região e ao pressuposto de independência entre as regiões. As medidas de correlação tendem a aumentar com as dimensões da unidade de análise escolhida. A agregação de dados em grandes regiões pode trazer uma falsa magnificação de associações entre variáveis agregadas (King, 1979). Por outro lado, a fragmentação da área de abrangência em um grande número de pequenas unidades espaciais de análise pode introduzir ruídos (confusão). Estatisticamente, existe um tamanho e uma divisão ótima da área de abrangência em unidades de análise que permitam: evidenciar diferenciais entre regiões, sem uniformizá-las; aumentar o poder estatístico de discernimento; e finalmente, estabilizar taxas de morbi-mortalidade, maximizando os valores do numerador e denominador (Morris \& $\mathrm{Mu}$ nasinghe, 1993). Por outro lado, se aceitarmos que eventos próximos não podem ser tomados como independentes, o uso de pequenas unidades de análise pode criar artificialmente uma alta correlação entre variáveis se estas tendem a ser similares em unidades territoriais próximas (King, 1979).

Novamente, a intensificação dos fluxos de trocas entre regiões impede que estas sejam vistas como autônomas. Para se estudar uma região deve-se compreender as relações, formas, funções, organizações e estruturas, que envolvem necessariamente a interrelação das forças presentes em diferentes escalas: local, regional, nacional e global (Santos, 1988).

\section{O espaço - lugar}

Uma terceira concepção de espaço é entendê-lo como um conjunto sem número de lugares, resultado da interação singular de grupos sociais com seu entorno mais próximo (Monken, 1995), ou, segundo Sabroza, a coexistência de grupos sócio-espaciais particulares que, apesar de compartilhar o mesmo espaço, possuem modos de vida diferentes em função de sua inserção social e cultura (Sabroza \& Leal, 1992). Dentro da mesma região, índios e garimpeiros possuem modos de vida, relacionados à sua inserção social, que produzem perfis epidemiológicos divergentes. Os lugares seriam na concepção de Milton Santos (1996), mesmo uma resistência ao processo de globalização e homogeneização do espaço.

A chamada Geografia Cultural tem destacado o papel das experiências individuais na formação do espaço e na inserção destes indivíduos neste espaço, enfatizando o comportamento e a cultura como determinantes das condições de saúde (Picheral \& Salem, 1992). Segundo a abordagem humanística da geografia médica, o espaço como mera localização deve ser substituído pelo lugar como espaço de experiência com padrão de troca e significado próprios (Gesler et al., 1997). Os limites da 
utilização desta abordagem encontram-se nas suas próprias premissas, que por considerar as singularidades do processo de produção de doenças, restringe seus resultados às condições específicas de cada lugar, não sendo reprodutíveis em outras condições sócio-ambientais.

Esta linha de trabalho na saúde utiliza os indicadores de saúde para uma melhor compreensão de um determinado território e da forma de produção de doenças. São exemplos as abordagens da antropologia médica desenvolvidas para a explicação da malária na Amazônia, e outras endemias no Brasil e África (Carmo, 1994; Norberg \& Finer, 1990). Neste caso, o conceito de espaço se aproxima da idéia de lugar, sendo resultado das singularidades das interações entre populações humanas e o ambiente. Seria o desligamento de grupos sociais de seus "lugares", ou seja, o processo de desterritorialização, um importante agente para a incorporação e propagação de riscos à saúde coletiva, através da intensificação de fluxos migratórios e desestruturação de redes sociais (Wallace, 1993). A África Subsahariana tem vivenciado nesta década epidemias quase simultâneas de Cólera, fome, Ebola, AIDS e violência exatamente numa região onde uma imensa massa humana se desloca em busca de água e áreas de menor conflito.

\section{O espaço do trabalho e saúde: a exposição ao mercúrio em uma fábrica de lâmpadas fluorescentes}

A dimensão espacial definida pelo trabalho, evidencia a relação direta entre as atividades humanas de natureza social e o espaço, que nesse contexto ganha uma dimensão além da geométrica e são constituídos lugares especiais, que podem ser modelados em estudos ecológicos de associação entre variáveis de exposição descritoras do processo de trabalho e indicadores biológicos de exposição.

Uma planta industrial é, necessariamente, um ambiente construído. Todo o espaço interno de uma indústria é planejado de modo a permitir fluxos de trabalho, energia, insumos, produtos e rejeitos que otimizem a produção de um determinado bem. $\mathrm{O}$ arranjo dos postos de trabalho é, portanto, preestabelecido em função da tecnologia disponível. No ambiente de trabalho industrial são reduzidas as variáveis e suas correlações são mais facilmente medidas e verificadas, sob menor efeito de interveniências confundidoras como ocorre nos lugares e regiões (Miettinen \& Cook, 1981). A escolha do exemplo da fábrica se adequa a este princípio simplificador do trabalho industrial taylorizado em linha de montagem.

O exemplo a seguir trata da produção de lâmpadas fluorescentes em três linhas de produção de uma indústria do Rio de Janeiro, envolvendo 110 trabalhadores distribuídos em três linhas paralelas de produção. Estas lâmpadas contêm no interior do cilindro de vidro cerca de $40 \mathrm{mg}$ de mercúrio metálico $(\mathrm{Hg})$. O fluxo de produção é mostrado na Figura 1. O recipiente de vidro é revestido com $\mathrm{CaPO}_{4}$ no setor "pintura" e encaminhado para o setor de "base", onde são montados, nas suas extremidades, os contatos elétricos. Estes contatos, por sua vez, são preparados no setor "montagem" e revistos no setor "swanson". No setor de ajuste de leds são armados os contatos e, após, ocorre a entrada da lâmpada na máquina de "esgotamento", onde é submetida a vácuo e é gotejada uma pequena quantidade de Hg. A lâmpada é então selada e submetida a "inspeção". Caso não apresente quebras ou defeitos nos contatos elétricos, as lâmpadas são novamente inspecionadas e embaladas. No caso de haver algum destes defeitos, a lâmpada é encaminhada para o setor "recuperação", onde os contatos elétricos são reaproveitados e reenviados para o setor "montagem".

Três principais focos de contaminação por Hg podem ser apontados, a princípio, ao longo do percurso do $\mathrm{Hg}$ como insumo e rejeito de produção. O primeiro situa-se nas atividades de preparo do recipiente gotejador de $\mathrm{Hg}$ ainda no laboratório da fábrica. Este recipiente é levado à máquina de esgotamento, que representa o segundo foco de contaminação. Um terceiro foco de suspeição é o setor de recuperação de lâmpadas quebradas, que contêm $\mathrm{Hg}$ no seu interior. A fábrica utiliza, segundo dados da produção, cerca de 833 g Hg.dia-1. O consumo de $\mathrm{Hg}$ no processo é de 800 g.dia-1, estimado com base na quantidade de lâmpadas produzidas (20.000 lamp.dia-1) e a massa de $\mathrm{Hg}$ presente em cada lâmpada. O montante de $\mathrm{Hg}$ disponibilizado (não necessariamente emitido) pela quebra de lâmpadas, cerca de $1 \%$ do total produzido, foi estimado em 8 g.dia-1 $^{-1}$ A maior parte do $\mathrm{Hg}$ potencialmente emitido na fábrica, cerca de 33 g.dia-1 é verificado ao longo do processo de estocagem, manipulação e gotejamento do Hg durante a produção de lâmpadas. Levantamentos realizados 
Figura 1

Fluxo de produção de lâmpadas fluorescentes e disposição espacial dos setores de trabalho. Os quadrados cheios representam a concentração média de $\mathrm{Hg}-\mathrm{U}$ (em $\left.\mu \mathrm{g} . \mathrm{l}^{-1}\right)$ nos diferentes setores da fábrica.

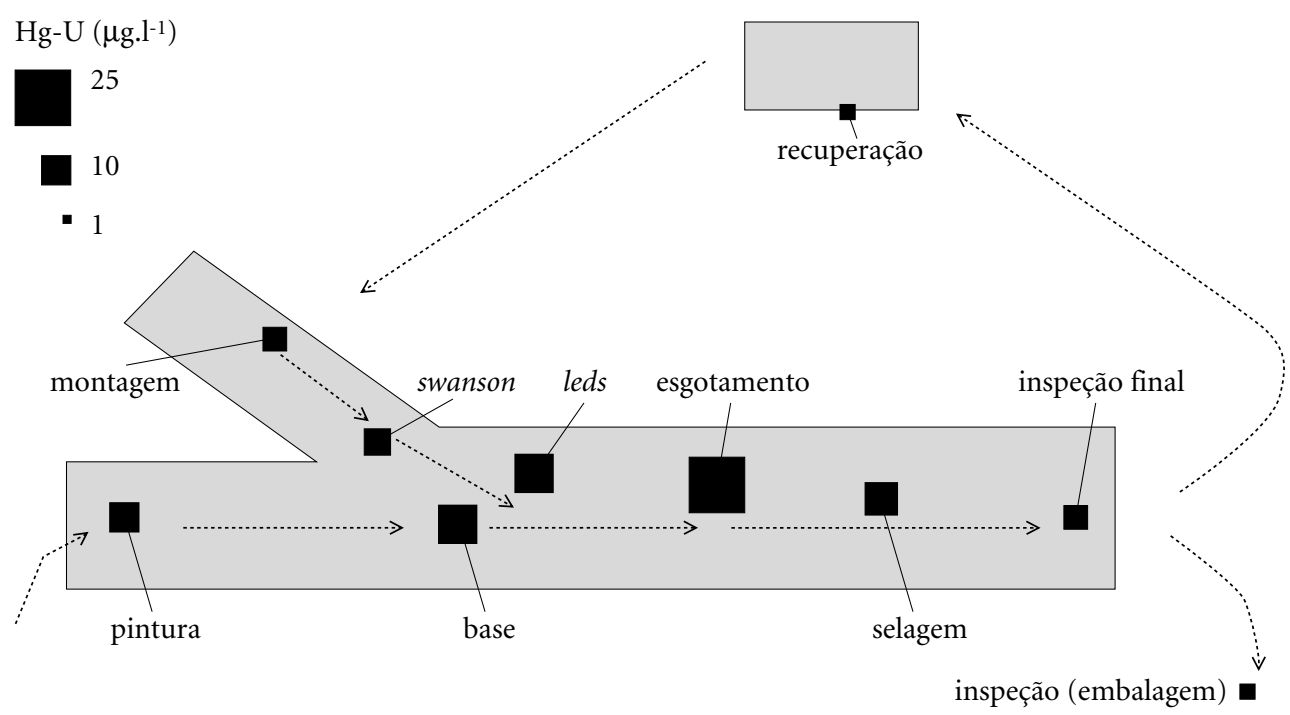

através de aparelhos de medição (eletrodos de fio de ouro), que indicam qualitativamente a presença de Hg metálico no ar, apontaram contaminação da máquina de esgotamento e, secundariamente, do local de recuperação de lâmpadas rompidas.

Foi realizada uma investigação de mercúrio urinário (Hg-U) em amostras colhidas em todos os funcionários da fábrica. Este parâmetro é um indicador da exposição recente dos trabalhadores a gases contendo mercúrio metálico (Minoia et al., 1990). Os dados de Hg$\mathrm{U}$ foram agregados e analisados segundo as categorias função, setor de trabalho e tempo de contratação.

A Tabela 1 apresenta os valores médios de $\mathrm{Hg}-\mathrm{U}$ agregados por função de trabalho na fábrica.

A reagregação de dados de $\mathrm{Hg}-\mathrm{U}$ por função permitiu a identificação de grupos laborais críticos, que apresentaram uma grande variação entre valores médios observados. As funções de analista e ajustador apresentaram as maiores médias de $\mathrm{Hg}-\mathrm{U}$ e são submetidas a exposição permanente ou esporádica ao $\mathrm{Hg}$ metálico. Estas são funções consideradas de alto grau de especialização, fortemente associadas com o tempo de trabalho dos funcionários na fábrica. Nestas funções enquadramse trabalhadores que operam máquinas sensí- veis, como a de esgotamento, ou que realizam inspeções ao longo da linha de produção, se expondo a agentes químicos e físicos de risco em momentos críticos da operação e manutenção da fábrica.

Foi verificada uma diferença de até uma ordem de grandeza entre os valores de $\mathrm{Hg}-\mathrm{U}$ nos diferentes setores de trabalho da fábrica. Diversos setores de produção apresentam como média valores considerados altos, considerando o limite máximo permissível para exposição ocupacional de $50 \mu \mathrm{g} . \mathrm{l}^{-1}$, representando uma exposição intensa dos trabalhadores ao Hg. Um destes setores é móvel, isto é, não possui postos de trabalho permanentes nas linhas de produção e é ocupado por trabalhadores com maior qualificação e tempo de trabalho, sendo responsáveis por atividades de inspeção e manutenção das linhas de produção. Por outro lado, alguns setores apresentam níveis próximos aos verificados em populações não expostas ao $\mathrm{Hg}$, de até $4 \mu \mathrm{g} . \mathrm{l}^{-1}$ (Minoia et al., 1990). A grande faixa de variação de Hg-U ao longo das linhas de produção pode indicar uma concentração de riscos em setores fisicamente delimitáveis e uma pequena mobilidade do Hg metálico no interior da fábrica. O vapor de $\mathrm{Hg}$ pode se deslocar, sem barreiras, por centenas de metros, tendo um tempo de residência na atmosfera de cerca de 
Tabela 1

Média de mercúrio urinário (Hg-U, em $\left.\mu \mathrm{g} . \mathrm{H}^{-1}\right)$ por função de trabalho na fábrica.

\begin{tabular}{lcc}
\hline Função & Hg-U ( $\boldsymbol{\mu g . l ^ { - 1 } )}$ & $\begin{array}{c}\text { número de } \\
\text { trabalhadores }\end{array}$ \\
\hline analista & 21,6 & 4 \\
ajustador & 17,6 & 23 \\
operador & 12,3 & 48 \\
líder & 9,8 & 5 \\
ajudante & 8,5 & 23 \\
supervisor & 3,8 & 1 \\
\hline
\end{tabular}

Tabela 2

Média de mercúrio urinário (Hg-U, em $\mu$ g.t-1) por setor de trabalho na fábrica.

\begin{tabular}{lcc}
\hline Setor & Hg-U ( $\boldsymbol{\mu g . \mathbf { l } ^ { - 1 } )}$ & $\begin{array}{c}\text { número de } \\
\text { trabalhadores }\end{array}$ \\
\hline esgotamento & 24,4 & 5 \\
móvel & 19,2 & 12 \\
leds & 16,9 & 4 \\
base & 16,6 & 12 \\
selagem & 14,9 & 4 \\
pintura & 12,3 & 12 \\
swanson & 11,3 & 4 \\
montagem & 10,3 & 12 \\
inspeção final & 9,5 & 28 \\
inspeção & 6,7 & 7 \\
recuperação & 6,4 & 2 \\
escritório & 3,8 & 1 \\
\hline
\end{tabular}

11 dias (Nriagu, 1979). Este não parece ser o caso do $\mathrm{Hg}$ nesta fábrica, que manipula $\mathrm{Hg}$ líquido, com volatilizacão localizada e limitada a poucos setores de trabalho.

A localização dos postos de trabalho e seu respectivo valor médio de $\mathrm{Hg}$-U é mostrada na Figura 1.

A exposição ao Hg afeta todos os trabalhadores diretamente envolvidos na produção de lâmpadas fluorescentes. Esta exposição se dá, no entanto, segundo um forte gradiente que aponta como fonte principal de contaminação a máquina de esgotamento. Setores considerados inicialmente suspeitos de contaminação, como o de recuperação de lâmpadas, apresentaram níveis inferiores a setores que não manipulam diretamente o $\mathrm{Hg}$, como o de pintura. A proximidade dos setores de pintura e selagem da fonte principal de contaminação aumenta consideravelmente a exposição destes trabalhadores ao Hg. Devido à dispersão dos vapores de $\mathrm{Hg}$ volatilizado, que permanece na atmosfera da fábrica, o nível de exposição de cada trabalhador está fortemente correlacionado à distância de seu posto de trabalho em relação à fonte principal de emissão de $\mathrm{Hg}$. A posição do posto de trabalho condiciona e está condicionado às demais categorias analisadas: função, setor e tempo de trabalho.

Não foi observada diferença significativa de níveis de $\mathrm{Hg}$-U entre trabalhadores das três linhas de produção, que apresentaram médias de 11,3 a 13,4 $\mu$ g.1-1. Também não foi verificada correlação linear significativa entre o nível de Hg-U e o tempo de trabalho dos funcionários na empresa $(r=0,06, n=104)$, apresentando uma pequena tendência de aumento da concentração de Hg na urina a cada ano de trabalho $\left(b=0,09 \mu \mathrm{g} \cdot 1^{-1} \cdot \mathrm{ano}^{-1}\right)$. Sendo o Hg um metal acumulativo no corpo humano, o tempo de trabalho, que envolve o tempo de exposição ao metal, pode ser um importante fator de risco para a saúde dos trabalhadores. $\mathrm{O} \mathrm{Hg}$, que possui uma meia-vida estimada no organismo de cerca de 60 dias (ASTDR, 1992), não reflete as exposições a longo prazo ao metal. Como setor e função são características mais estáveis do trabalhador que o nível de $\mathrm{Hg}-\mathrm{U}$, tendo os trabalhadores da fábrica mais de dois anos em média em cada setor de trabalho, esta variável irá se relacionar indiretamente com setor e função.

Por outro lado, o tempo de trabalho está relacionado ao grau de especialização dos trabalhadores. O tempo de trabalho pode ser menor em setores de risco, onde as condições de insalubridade, ou a agressividade do processo de trabalho, aumentem a rotatividade de funcionários na atividade, como observado em uma indústria metalúrgica em locais com exposição à arsina $\left(\mathrm{AsH}_{3}\right)$ (Machado et al., 1992).

A Figura 2 mostra as possíveis relações entre fatores que podem influir na exposição global dos trabalhadores ao Hg. O grau de exposição ao mercúrio está correlacionado à função exercida e ao setor de trabalho dos funcionários da fábrica. Por outro lado, tempo de trabalho, função e setor estão associados devido à organização interna do trabalho na fábrica. Esta organização do trabalho se reflete na posição de trabalho e organização espacial da produção na fábrica. Algumas destas relações podem ser verificadas estatisticamente. Ao se estudar a epidemiologia da exposição é necessário estabelecer o interrelacionamento entre o espaço e as pessoas (Briggs, 1992) que 


\section{Figura 2}

Relações entre organização do trabalho, organização espacial de produção e exposição ao Hg em uma fábrica de lâmpadas fluorescentes.

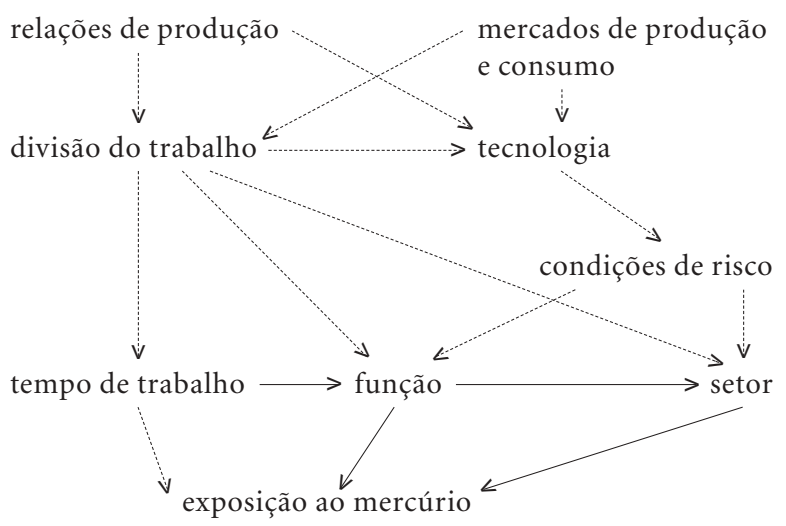

relações presumidas.

associações verificadas neste trabalho.

no caso da contaminação ocupacional é realizada pela função/atividade e setor/atividade de trabalho. A localização das pessoas no ambiente da fábrica é determinada pelo setor de trabalho, enquanto a função reflete o grau de mobilidade e qualificação de uma ocupação na população. Outras relações, não menos importantes, dizem respeito à organização de produção, que pelo seu caráter singular, pode ser apenas estudada através da análise qualitativa do processo de produção e saúde.

Toda a linha de produção de lâmpadas aqui descrita é voltada para o mercado externo à fábrica. Alterações estruturais e conjunturais no mercado de produção e de consumo de lâmpadas, além do mercado de trabalho, podem modificar consideravelmente as condições tecnológicas e de trabalho. As condições de risco estão, portanto, subordinadas também à lógica externa de produção.

\section{Considerações finais}

As análises em saúde têm utilizado o espaço através de diferentes abordagens, resultado da concepção diversificada do próprio conceito de espaço. Talvez por isso, o uso do espaço como categoria de análise permita, mais que a identificação de fatores causais, o estabelecimento do contexto social e ambiental em que se processam os fenômenos de saúde. O geoprocessamento pode ser utilizado como ferramenta de organização e análise de dados sobre saúde e ambiente, permitindo uma melhor visualização do contexto em que estas relações ocorrem, bem como o aperfeiçoamento dos próprios sistemas de informação. A vigilância em saúde não pode prescindir deste instrumento de organização e análise de dados primários e provenientes dos sistemas de informação hoje implantados.

Na concepção da geografia social, o espaço é transformado em função das atividades humanas de produção e reprodução, sendo o trabalho classicamente ligado às atividades de produção. O fenômeno de globalização traz consigo um modelo hegemônico de relações de trabalho e reprodução social, com o crescimento do setor de serviços e especialmente as atividades ligadas ao transporte, lazer e informação. As atividades laborais, no entanto, continuam a exercer impacto no ambiente e são, em si, organizadoras da sociedade e do espaço. O deslocamento do eixo de resistências para fora da fábrica, com uma crescente importância dos movimentos ecológicos não invalida e sim se agrega ao movimento operário a força perdida pela transformação das relações de trabalho, com a terceirização, precarização e desemprego industrial. Neste cenário, uma questão fundamental das relações entre espaço e saúde é a maior repercussão dos ambientes industriais sobre saúde dos trabalhadores do terceiro mundo e nos estratos de trabalhadores com menos direitos sociais (Porto \& Freitas, 1996).

As conclusões do estudo discutido no exemplo se restringem ao domínio de uma fábrica, com condições tecnológicas, de organização de trabalho e ambientais singulares. Dificilmente duas fábricas possuem arranjos espaciais e contornos sociais semelhantes. A metodologia utilizada neste trabalho permite, no entanto, o entendimento de condições de risco que se podem reproduzir em outras situações, sobre as quais intervêm fatores relacionados não só à tecnologia implantada como a organização - social e espacial - do trabalho. Esta estrutura tem reflexos óbvios sobre as condições ambientais de risco.

Os estudos espaciais permitem sua reprodução de forma proporcional à escala de análise, sendo os achados moleculares universais (Susser, 1995). Os estudos de exposição em ambientes fabris têm, portanto, uma reprodu- 
tibilidade de resultados maior que os realizados em escala regional, daí a validade de sua utilização destes ambientes como ensaios sobre a relação entre os lugares e as pessoas. A fábrica pode ser tomada como exemplo extremo de organização espacial, onde cada posto de trabalho foi planejado em função da distribuição de operações e máquinas ao longo da linha de produção. Utilizando metodologia semelhante um levantamento realizado em crianças de Santo Amaro (Bahia) mostrou que a distância dos domicílios às fontes de contaminação ambiental é o principal fator de risco para a intoxicação por chumbo. Esta variável, no entanto, está fortemente associada a aspectos da urbanização e industrialização da região, isto é, condicionada à própria organização espacial da cidade (Silvany-Neto, 1982). Uma cidade ou região possuem graus maiores de autonomia, que permitem a produção do espaço e sua organização espacial através de outros agentes modeladores, como o estado, as organizações sociais e a própria paisagem natural. A transposição desses exemplos para a escala regional ou nacional é perigosa por não prever o próprio espaço como agente de transformação da vida cotidiana e da macroorganização espacial e econômica deste território. Por outro lado, como se trata de um espaço concebido para e pela produção, a fábrica permite estudar as múltiplas relações entre a organização da sociedade e do espaço. Desta organização resultam condições específicas de risco que podem afetar a saúde da população a ela submetida.

Entre os fatores de risco intervenientes na produção de doenças encontram-se variáveis de natureza ambiental, cultural, social e genética. Essas variáveis encontram-se, muitas vezes, correlacionadas. Padrões de comportamento de um grupo social podem determinar uma série de situações de exposição, concentrando ou excluindo um grande número de fatores de risco. Esta conjunção de riscos pode ser ainda mais marcante nos agregados espaciais. As regiões muitas vezes, pela própria definição, reúnem padrões hegemônicos de etnia, hábitos alimentares, tipos de solo, atividades agrícolas, clima, organização social etc. Uma região, segundo a concepção de Vidal de la Blache corresponde a uma relação entre a sua população e o ambiente, o que lhe confere singularidade (Corrêa, 1986). Esta abordagem foi intensamente criticada por correntes da geografia posteriores a La Blache mas pa- rece que volta a tona com os geógrafos chamados pós-modernos (Soja, 1993). Fatores culturais, econômicos, demográficos e ambientais estão presentes em todas as escalas em que se represente o espaço. A forma ou estratégia com que se divide o território não lhe confere homogeneidade. Como afirma Dorfman (1995), a opção pela escala de análise é um processo de "esquecimento coerente", um recorte no conjunto dos elementos reais, de acordo com os fins. Mesmo as chamadas "regiões geográficas” possuem diferenciais internos importantes de renda, etnia, hábitos alimentares etc. O desenho de unidades geográficas parece ser assumidamente artificial, baseado em critérios políticos, ambientais, e antes de mais nada operacionais. Deste modo, a concepção da região como área homogênea "baseia-se na delimitação de um território a partir da uniformidade de certas características", em detrimento de outras, onde os critérios e objetivos de trabalho indicarão as variáveis a serem utilizadas para regionalização (Piquet et al., 1986).

No caso do geoprocessamento, a escolha da escala de trabalho se delineia com o estabelecimento a priori das unidades de agregação de dados e da extensão do território de trabalho (Barcellos \& Santos, 1997). Além de restringir sua abrangência, a escala de análise condiciona os estudos em saúde e ambiente fornecendo maior ou menor peso a fatores sociais, ambientais e econômicos. Isto se dá exatamente porque de uma para outra escala mudam as unidades geográficas e seus conteúdos. No exemplo da fábrica de lâmpadas fluorescentes, a unidade espacial de análise é o posto de trabalho. A análise dos diferenciais de risco entre postos de trabalho envolve necessariamente a organização espacial e social da produção, um determinante de saúde que é evidenciado nesta escala de análise.

O exemplo apresentado demonstra uma concepção metodológica, de construção de um modelo de relações entre variáveis qualificadoras e quantificadoras da exposição a um determinado agente potencialmente patogênico de interesse para saúde. O modelo visto em sua dinâmica oferece uma possibilidade de avaliação das alterações em seus componentes e uma aprofundamento do entendimento dos resultados, em um processo de aproximação sucessiva da explicação das diferenças internas aos estratos de cada variável componente do modelo. 


\section{Referências}

ASTDR - Agency for Toxic Substances and Disease Register. 1992. Case Studies in Environmental Medicine: Mercury Toxicity. V.17, Chicago

Avila-Pires F 1983. Princípios de Ecologia Humana. Ed. da Universidade Federal do Rio Grande do Sul, Porto Alegre. 158 pp.

Bailey TC \& Gratell AC 1995. Interactive Spatial Data Analysis. Longman Scientific \& Technical, Essex, England.

Barcellos C \& Bastos FI 1996a. Redes sociais e difusão da AIDS no Brasil. Boletim de la Oficina Sanitaria Panamericana. 121(1): 11-24.

Barcellos C \& Bastos FI 1996b. Geoprocessamento, ambiente e saúde, uma união possível? Cadernos de Saúde Pública 12(3): 389-397.

Barcellos C \& Santos SM 1997. Colocando dados no mapa: a escolha da unidade de agregação e integração de bases de dados em saúde e ambiente através do geoprocessamento. Informe Epidemiológico do SUS VI(1): 21-29.

Breilh J 1991. Epidemiologia, Economia, Política e Saúde. Ed. Hucitec, São Paulo.

Briggs DJ 1992. Mapping environmental exposure, p. 158-176. In P Elliot, Geographical and Environmental Epidemiology: Methods for Small-area Studies. Oxford University Press, Tokyo.

Carmo EH 1994. Esquitossomose Mansônica no Estado da Bahia: Produção da Doença no Espaço e Medidas de Controle. Dissertação de Mestrado. Faculdade de Medicina, Universidade Federal da Bahia, Salvador.

Carvalho MS 1997. Aplicação de Métodos de Análise Espacial na Caracterização de Áreas de Risco à Saúde. Tese de Doutorado. Coordenação de Programas de Pós-graduação em Engenharia, COPPE/UFRJ, Rio de Janeiro.

Castro MC 1996. Geoprocessamento. Geografia Quantitativa. De volta para o futuro? p. 19-31. 1a Semana Estadual de Geoprocessamento. Forum Estadual de Geoprocessamento do Rio de Janeiro, Rio de Janeiro.

Corrêa RL 1986. Região e Organização Espacial. Ed. Ática, São Paulo. 93 pp.

Dorfman A 1995. As escalas do território e sua articulação: uma revisão, p. 99-108. In Z Mesquita \& CR Brandão (orgs.) - Territórios do Cotidiano. Ed. Universidade, Porto Alegre.

Duchiade M P 1991. Mortalidade Infantil por Pneumonia na Região Metropolitana do Rio de Janeiro, 19761986. Tese de Mestrado. Escola Nacional de Saúde Pública, Rio de Janeiro.

Gesler WM, Bird ST \& Oljeski SA 1997. Disease ecology and a reformist alternative: the case of infant mortality. Social Science \& Medicine 44(5): 657-671.

Godin PM, Feinberg MH \& Ducauze CJ 1985. Modelling of soil contamination by airborne lead and cadmium around several emission sources. Environmental Pollution (Series B) 10: 97-114.

Haggett TP 1975. Geography: a Modern Synthesis. 2nd ed. Ed. Harper \& Row, New York.

Hills M \& Alexander F 1989. Statistical methods used in assessing the risk of disease near a source of possible environmental pollution: a review. Journal of Royal Statistical Society Association. 152: 353-363
Hugh-Jones M 1989. Application of remote sensing to the identification of the habitats of parasites and disease vectors. Parasitology Today 5(8): 224-251

Hutt MSR \& Burkitt DP 1986. The Geography of Noninfectious Diseases. Oxford University Press, Oxford. $164 \mathrm{pp}$.

King PE 1979. Problems of spatial analysis in geographical epidemiology. Social Science \& Medicine 13: 249-252.

Machado JH, Barcellos C, Lima LQ \& Pivetta F 1992. Arsenic exposure in the working environment of a zinc smelting plant - homogeneous group analisys. p. 153-158. International Seminar Arsenic in the Environment and its Incidence on Health. Santiago de Chile.

Miettnem OS \& Cook EF 1981. Confounding: essence and detection. American Journal of Epidemiolology 114: 553-603.

Minoia C, Sabbioni E, Apostoli P 1990. Trace element reference values in tissues from inhabitants of the European Community. A study of 46 elements in urine, blood and serun of healthy italian subjects. The Science of the Total Environment 95: 89-105.

Monken M 1995. A noção de Lugar e o Estudo da Realidade na Ilha Grande (RJ). Instituto de Planejamento Urbano e Regional, IPPUR/UFRJ.

Morris RD \& Munasinghe RL 1993. Aggregation of existing geographic region to diminish spurious variability of disease rates. Statistics in Medicine 12:19151929.

Norberg E \& Finer D 1990. Society, Environment and Health in Low-income Countries. Karolinska Institutet, Goteborg.

Nriagu JO 1990. The Biochemistry of Mercury in the Environment: Topics in Environmental Health. Ed. Elsevier, Amsterdam.

Openshaw S, Charlton M, Wymer C \& Craft A 1987. Building a Mark 1 geographical analysis machine for the automated analysis of point pattern cancer and other spatial data. Research report, 12. Economic and Social Research Council, Northern Regional Research Laboratory, University of Newcastle upon Tyne, Newcastle, UK.

Paim JS 1997. Abordagens teórico-conceituais em estudos de condições de vida e saúde: notas para reflexão e ação, p. 7-30. In RB Barata (org.) - Condições de Vida e Situação de Saúde. Abrasco, Rio de Janeiro.

Picheral H \& Salem G 1992. De la Géographie Medicale à la Géographie de la Santé. Bilan et Tendances de la Géographie Française (1960 - 1991). Cahiers Geo 22, Montpellier

Piquet R, Randolph R, Smolka M \& Vetter D 1986. Análise das Articulações Sócio-econômicas Regionais: Sugestões Metodológicas. Programa de Pós-Graduação em Planejamento Urbano e Regional. IPPUR/ UFRJ, Universidade Federal do Rio de Janeiro, Rio de Janeiro.

Porto MFS \& Freitas CM 1996. Major chemical accidents in industrializing countries - the socio political amplification of risks. Risk analysis 16(1): 19-29.

Sabroza PC \& Leal MC 1992. Saúde, ambiente e desenvolvimento: alguns conceitos fundamentais, p. 4593. In MC Leal, PC Sabroza, RH Rodriguez \& PM 
Bus (orgs.) - Saúde, Ambiente e Desenvolvimento. vol. 1. Ed. Hucitec-Abrasco, São Paulo.

Santos M 1988. Espaço e Método. Ed. Nobel, São Paulo. Santos M 1996. Metamorfoses do Espaço Habitado. Ed. Hucitec, São Paulo.

Silvany Neto R 1982. Urbanização e Poluição Industrial: Determinação Social da Intoxicação Pelo Chumbo Em Crianças de Santo Amaro - Bahia. Tese de Mestrado. Universidade Federal da Bahia, Salvador.

Soja EW 1993. Geografias Pós-modernas: a Reafirmação do Espaço na Teoria Social Crítica. Ed. Jorge Zahar, Rio de Janeiro.

Stephens C, Timaes I, Akerman M, Avle S, Maia PB, Campanario P, Doe B, Lush L, Tetteh D \& Harpham T 1994. Collaborative Study in Accra, Ghana and São Paulo, Brazil - Analysis of Urban Data of Four Demographic and Health Surveys. London School of
Hygiene \& Tropical Medicine, London.

Susser M 1995. Conferência, Anais do 3 Congresso Brasileiro de Epidemiologia, Salvador.

Szwarcwald CL \& Leal MC 1997. Estatística espacial na análise exploratória de dados epidemiológicos, p. 251-270. In RB Barata (org.) - Condições de Vida e Situação de Saúde. Ed. ABRASCO, Rio de Janeiro.

Wallace R 1993. Social desintegration and the spread of AIDS. Social Science \& Medicine 38(7): 887-896.

Wartenberg D, Greenberg M \& Lathrop R 1993. Identification and characterization of populations living near high-voltage transmission lines: a pilot study. Environmental Health Perspectives 101: 626-632.

Werneck GL \& Struchiner CJ 1997. Estudos de agregados de doenças no espaço-tempo: conceitos, técnicas e desafios. Cadernos de Saúde Pública 13(4): 611-624. 